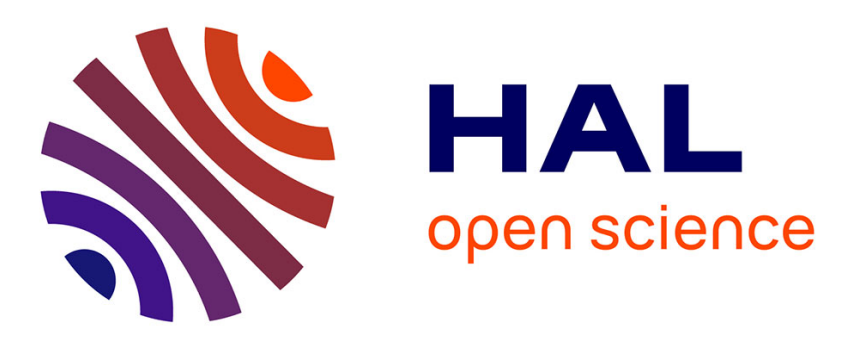

\title{
Dieu, le Pape et la Sainte Vierge: un mouvement de contestation de l'Eglise catholique au Bénin
}

\author{
Emmanuelle Kadya Tall
}

\section{To cite this version:}

Emmanuelle Kadya Tall. Dieu, le Pape et la Sainte Vierge: un mouvement de contestation de l'Eglise catholique au Bénin. Collective mobilisations in Africa / Mobilisations collectives en Afrique, Brill, pp.91-111, 2015, 9789004293175. hal-01435657

\section{HAL Id: hal-01435657 https://hal.science/hal-01435657}

Submitted on 19 Jan 2017

HAL is a multi-disciplinary open access archive for the deposit and dissemination of scientific research documents, whether they are published or not. The documents may come from teaching and research institutions in France or abroad, or from public or private research centers.
L'archive ouverte pluridisciplinaire HAL, est destinée au dépôt et à la diffusion de documents scientifiques de niveau recherche, publiés ou non, émanant des établissements d'enseignement et de recherche français ou étrangers, des laboratoires publics ou privés. 


\title{
Dieu, le Pape et la Sainte Vierge : \\ un mouvement de contestation de l'Église catholique au Bénin.
}

\author{
Emmanuelle Kadya Tall
}

À partir de la fin 2008, dans une commune du centre méridional du pays, Banamè, un prêtre exorciseur catholique délivre successivement deux jeunes filles de maux qu'il attribue à l'esprit malin. La première, Nicole, souffre de troubles de la vue, la seconde, Parfaite, souffre de troubles du comportement. Dès 2009, ces deux jeunes filles appelées familièrement les « petites filles », vont devenir sous l'emprise du prêtre exorciste, les réceptacles de la Sainte Vierge pour Nicole et de Dieu pour Parfaite. Les révélations de Dieu sont de plus en plus radicales et ses blâmes stigmatisent une société considérée comme gangrenée de haut en bas par la sorcellerie. Les accusations visent principalement le clergé et les autorités politiques. Ces diatribes vont éloigner progressivement plusieurs alliés. En premier lieu le clergé qui semblait d'emblée accueillir avec bonheur les dons révélés des deux jeunes filles. Ensuite un nombre grandissant de fidèles qui soupçonnent une escroquerie au vu de l'enrichissement rapide et ostentatoire des premiers membres de cette nouvelle communauté ecclésiastique.

En revanche, les prêches de Banamè attirent de plus en plus de déçus du Renouveau démocratique $^{1}$. Parmi ces mécontents ${ }^{2}$, une population essentiellement urbaine et jeune, parfois hautement diplômée et, dans tous les cas, frustrée dans ses aspirations sociales et souvent lassée du jeu électoral corrompu qui ne laisse pas de place au renouvellement du corps politique ${ }^{3}$. En effet, népotisme et clientélisme prévalent encore et toujours sur les qualifications professionnelles, ce qui accroît la frustration, d'autant que, grâce aux nouvelles technologies, des images, quasi instantanées et permanentes, circulent sur lesquelles s'affichent la puissance et la prospérite des gouvernants et de l'élite. À portée du regard de tous, elles exacerbent le désir pour chacun d'avoir sa part du gâteau.

\footnotetext{
${ }^{1}$ Le Renouveau démocratique est l'appellation donnée au retour à la démocratie après la Conférence nationale des forces vives de la nation de janvier-février 1990.

2 Cette expression fait référence aux «mécontents de la modernité » dont traite l'ouvrage édité par Jean Comaroff et John Comaroff, Modernity and its Malcontents: Ritual and Power in Postcolonial Africa (Chicago: The University of Chicago Press, 1993).

${ }^{3}$ Voir Cédric Mayrargue « Les élites politiques béninoises au temps du Renouveau démocratique : entre continuité et transformation », dans Le non renouvellement des élites en Afrique subsaharienne, dir. Jean-Pascal Daloz et al. (Bordeaux : Centre d'étude d'Afrique noire, 1999) : 33-56.
} 
Pourquoi s'intéresser à un mouvement de contestation religieuse chrétienne sans recul historique et comment renouveler l'analyse des christianismes africains, dès lors que le phénomène de prolifération des mouvements prophétiques et messianiques en Afrique subsaharienne a déjà fait l'objet de nombreuses analyses depuis les premiers temps de la confrontation entre Européens et Africains ${ }^{4}$ ? Comment renouveler cette compréhension en s'appuyant sur une situation où le religieux s'articule à plusieurs autres secteurs de la société-: la finance, le politique et la sorcellerie ?

Au-delà des explications en termes de réaction aux bouleversements socio-historiques induits par la traite atlantique, les conquêtes coloniales, les processus de décolonisation et d'indépendance ou encore en termes de mobilisation contre les régimes autoritaires d'inspiration marxiste, que peut-on dire d'un mouvement qui émerge dans un pays qui a su passer d'un régime despotique à un régime démocratique sans violence, contrairement à certains pays de la sous-région ? Comment vingt ans de démocratisation, de pluralisme et de décentralisation peuvent-ils faire le nid d'un nouveau mouvement réformiste dans un pays où, il y a un peu plus de soixante ans, était fondée l'une des plus importantes Églises africaines, l'Église du christianisme céleste ${ }^{5}$ qui rayonne bien au-delà de la sous-région ? Mais il faut aussi rappeler, et ce n'est pas anodin, que le Bénin tant aimé des Dieux, comme se plaît à le clamer l'opinion publique, est également la terre du catholicisme où ce sont des prêtres comme le père Aupiais ${ }^{6}$ durant la colonisation, et plus récemment encore le cardinal de Souza, lors de la Conférence nationale de 1990, qui ont ouvert les esprits à la pluralité religieuse et politique, en évitant de stigmatiser les acteurs des autres religions. À l'heure où le renouveau évangélique frappe de plein fouet les églises historiques, il nous semble particulièrement bienvenu de nous intéresser à la mobilisation de catholiques contre l'Église romaine au Bénin.

Ce qui caractérise de prime abord le mouvement de Banamè, c'est, d'un côté, qu'il se revendique comme mouvement réformiste catholique romain, à l'inverse des réformismes chrétiens contemporains en grande majorité d'obédience néo-pentecôtiste et, de l'autre, qu'il

\footnotetext{
${ }^{4}$ Les mouvements prophétiques et messianiques précoloniaux (la prophétesse Kimpa Vita et les antoniens au $\mathrm{XVII}^{\mathrm{e}}$ et $\mathrm{XVIII}^{\mathrm{e}}$ siècle en Afrique centrale), les mouvements prophétiques qui ont accompagné d'abord les colonisations européennes à partir de la fin du XIX siècle et ensuite les processus de décolonisation ont tous en commun d'être des mouvements en réaction aux transformations sociales en profondeur des sociétés locales.

${ }^{5}$ Pour plus de détail sur cette Église, voir Christine Henry, La force des anges : rites, hiérarchie et divination dans le Christianisme Céleste (Bénin) (Turnhout, Brepols : Bibliothèque de l'École des hautes études, Sciences religieuses, vol. 135, 2008).

${ }^{6}$ Dans son article G. Ciarcia souligne comment le père Aupiais, avec sa sensibilité anthropologique qui rompt avec le comportement de ses prédécesseurs, est en quelque sorte l'initiateur d'une certaine forme de mise en patrimoine de ce qu'il appelle le cérémonialisme qu'il considère être en syntonie avec la morale chrétienne. Voir Gaetano Ciarcia, «Réthoriques et pratiques de l'inculturation: une généalogie "morale” des mémoires de l'esclavage au Bénin », Gradhiva 8 (2008) : 30.
} 
s'agit d'un mouvement qui naît dans un contexte non autoritaire, contrairement à ce que pouvait être celui de la colonisation pour la plupart des Églises africaines ou un régime militaire si l'on se réfère à l'Église universelle du royaume de Dieu, une Église évangélique fondée au Brésil en $1977^{7}$. On soulignera d'ailleurs que cette Église, qui tire son succès en grande partie de ses méthodes de communication, ne s'est jamais implantée au Bénin, ce qui est loin d'être anodin. La fondation de l'Église du christianisme céleste a sans aucun doute découragé ses velléités d'affronter une terre pourtant fameuse pour ses sorciers. En effet, la spécificité de l'Église brésilienne est d'attribuer une couleur locale aux maux de chaque nation, en opérant un travail de traduction des ordres symboliques locaux pour donner corps aux différentes figures du démon et de ses acolytes. En diabolisant entités, techniques et savoirs vernaculaires, les génies des eaux en Côte d'ivoire, les marabouts guérisseurs au Sénégal, les pasteurs lusophones désignent d'emblée un univers symbolique avec sa myriade d'entités souvent rapportées à un monde païen auquel est également associé le catholicisme en raison de son culte des saints.

Néanmoins, comme dans la plupart des mouvements néo-pentecôtistes, c'est sur le terrain de la sorcellerie que s'appuie ce mouvement de contestation de l'Église romaine : le prêtre exorciste va être à l'origine, avec l'aide de ses deux médiums, les dites fillettes Parfaite et Nicole, d'un phénomène qui très vite va retenir l'attention d'une population avide de miracles. Mais avant de décrire ce mouvement, revenons un instant au contexte de son émergence.

\section{Dieu aime le Bénin : Renouveau démocratique et pluralisme religieux}

Rappelons que c'est la Conférence nationale qui est à l'origine de l'adage théologique « Dieu aime le Bénin ». Les raisons en sont que cela a été le premier et seul événement politique qui a instauré le multipartisme sans effusion de sang sur le continent subsaharien, contrairement à d'autres conférences nationales qui se sont ttransformées en de violents conflits (Congo Brazzaville), ou encore, qui ont reconduit des régimes autoritaires (Togo). Au Bénin, la Conférence s'est déroulée pacifiquement et a véritablement ouvert la voie à des processus de démocratisation et de décentralisation du pouvoir d'État.

\footnotetext{
${ }^{7}$ L'Église universelle du royaume de Dieu fait du prosélytisme dans de nombreux pays africains et capitales européennes, voir André Corten, Jean-Pierre Dozon et Ari Pedro Oro, Les nouveaux conquérants de la foi : l'Église universelle du royaume de Dieu, Brésil (Paris : Karthala, 2003).
} 
L'adage « Dieu aime le Bénin » réconforte un tant soit peu une population désabusée face à l'accroissement des inégalités entre nantis et démunis et aussi à la prévalence du népotisme et du régionalisme. Si la corruption peut sembler inhérente aux démocraties, qu'elles soient africaines ou européennes, la politique du ventre ${ }^{8}$ ne suffit pas à elle seule à expliquer le fonctionnement politique en Afrique, en particulier depuis que la dé-légitimation de la grande alternative capitalisme $v s$ socialisme a ouvert une voie royale aux mobilisations identitaires et religieuses.

Ainsi au Bénin, après la disette imposée par le programme d'ajustement structurel exigé par le FMI (Fonds monétaire international), lors de la fin de «règne» de Mathieu Kérékou (1972-1990), alors encore «marxiste-léniniste» et autoritaire, la manne internationale des pays du nord a déferlé sur le pays, avec l'idée que chaque citoyen puisse se transformer en auto-entrepreneur via la création d'organisations non gouvernementales. Durant les premières années du Renouveau démocratique, nombreux chefs de culte et représentants des autorités traditionnelles ont tenté de développer leur entreprise de pourvoyeurs de biens magico-religieux et culturels pour une clientèle diasporique issue du nouveau monde avec plus ou moins de succès ${ }^{9}$. La manne internationale s'étant tarie à partir du troisième millénaire, c'est dans la financiarisation des banques et des sociétés de crédit que certains Béninois ont espéré trouver le salut en s'enrichissant. La proximité entre certains hauts membres des Églises de réveil (Christianisme céleste et mouvements évangéliques) et certaines sociétés de crédit comme ICC Services témoigne des liens étroits qui existent entre salut et manne financière.

Différents cas illustrent cet enchevêtrement d'univers sociaux. En effet, si l'on considère la renaissance de Kérékou en $\mathrm{Christ}^{10}$ en pleine Conférence nationale (19-28 février1990), suivie quelques mois plus tard par l'affection mystérieuse de Soglo lors de la campagne présidentielle (maladie soignée par l'ancien roi de Kétou, grand connaisseur des sciences vodun) et l'instauration d'un jour férié dédié aux cultes vodun quelques années plus tard (1996), force est de constater que multipartisme et pluralisme religieux sont étroitement intriqués. Le pluralisme religieux met en présence les religions révélées, les cultes vodun et

\footnotetext{
${ }^{8}$ Voir Jean-François Bayart. L'État en Afrique : la politique du ventre (Paris : Fayard « L'espace du politique », 1989).

${ }^{9}$ E. Kadya Tall, «Stratégies locales et relations internationales des chefs de culte au Sud-Bénin», dans Entreprises religieuses transnationales en Afrique de l'Ouest, dir. Laurent Fourchard, André Mary et René Otayek. Paris : Karthala (2005) : 267-284.

${ }^{10}$ Pour plus de détails sur la conversion de Kérékou et son parcours théologico-politique voir Camilla Strandsbjerg, «Continuité et rupture dans les représentations du pouvoir politique au Bénin entre 1972-2001 : le président Kérékou, du militaire-marxiste au démocrate pasteur », Cahiers d'études africaines 177 (2005) : http://etudesafricaines.revues.org/4903.
} 
l'explication de tous les malheurs et infortunes en termes sorcellaires. En raison des nombreuses institutions de protection contre la sorcellerie et la diablerie actives dans le pays, on pourrait facilement être amené à dire que « Dieu aime le Bénin ».

Ce pluralisme vient réinterpréter la dimension théologico-politique du régime « marxiste » de Kérékou qui a été largement établie, tout comme l'importance du pouvoir de la véranda dans les États postcoloniaux africains ${ }^{11}$. Décliné en termes de force mystique, sorcellerie et magie, ou encore de pouvoir ou forces occultes par les chercheurs en sciences sociales $^{12}$, la dimension cachée du pouvoir politique est bien souvent traduite en termes appartenant au vocabulaire théologique. La référence au symbolisme religieux est ainsi toujours marquée dans les emblèmes des chefs d'État ou dans ceux de leur parti : de la canne sceptre avec un caméléon sculpté et du col Mao de Kérékou durant sa période révolutionnaire à sa tunique soutane d'homme re-né (born again) devenu prophète, à la jarre trouée de la coalition l'Union fait la nation (UN) ou encore le cauris des Forces cauris pour un Bénin émergent (FCBE) de la mouvance présidentielle lors des élections de 2011, il est important de souligner que tous ces emblèmes évoquent, mettent en scène ou, pour reprendre la terminologie marinienne ${ }^{13}$, re-présentent, i.e. mettent en présence réelle un pouvoir qui s'exerce dans sa représentation. Et cette représentation est théologique au sens où ces images appartiennent au vaste champ de la mystique vodun: le don de métamorphose pour le caméléon, mais également le signe de la part féminine (Lisa) de la divinité suprême de la cosmologie vodun - évocation de la prospérité du royaume du Danxomé durant le règne de Guezo $^{14}$; quant au cauris, ancienne monnaie d'échange durant la période précoloniale, il est également un instrument de la géomancie $\mathrm{Fa}^{15}$, technique permettant de prévoir le futur pour en corriger les mauvais effets pour le consultant.

\footnotetext{
${ }^{11}$ Voir Richard Banégas, La démocratie à pas de caméléon: transition et imaginaires politiques au Bénin. (Paris : Karthala, 2003) et Strandsbjerg, « Continuité et rupture », pour le Bénin, et pour la métaphore du pouvoir du climatiseur et de la véranda, voir Emmanuel Terray, " Afrique ambiguë : le climatiseur et la véranda », dans Afrique plurielle, Afrique actuelle. Hommage à Georges Balandier (Paris : Karthala, 1986) : 37-44.

${ }^{12}$ Cf. Comaroff, dir., Modernity and its Malcontents, Peter Geschiere, Sorcellerie et politique en Afrique : la viande des autres (Paris : Karthala. 1995), Birgit Meyer, «The power of money : politics, occult forces, and Pentecostalism in Ghana », African Studies Review 41 n $^{\circ} 3$ (1998) : 15-37.

${ }^{13}$ Voir la définition de Louis Marin, « Baroque, Classique : Versailles ou l'architecture du Prince », dans Politiques de la représentation. Paris : Kimé, 2005 : 247 -261.

${ }^{14}$ Guezo régna de 1818 à 1858 et enrichit son royaume grâce aux richesses provenant de la traite atlantique. Cependant, conscient de la fin de l'esclavage, sur les conseils de son ami Chacha qu'il fera vice-roi de Ouidah, il amorça la transformation de l'économie esclavagiste en économie de plantation pour faire face à la prohibition européenne de l'esclavage. Voir Bruce Chatwin, The Viceroy of Ouidah (London : Jonathan Cape, 1980). On notera cependant que la traite interlope se poursuivit avec le Brésil qui n'abolit l'esclavage qu'en 1888.

${ }^{15}$ Voir Bernard Maupoil, La géomancie à l'ancienne Côte des Esclaves (Paris : Institut d'ethnologie, 1981 [1943]).
} 
Toujours pour conforter la réflexion sur l'articulation du renouveau démocratique et du pluralisme religieux, il importe de noter, comme l'a fait Issaka K. Souaré, qu'au Bénin, depuis le multipartisme, aucun candidat à la présidence ayant gagné les élections, n'a été porté par un parti ${ }^{16}$. Cependant, on ne peut ignorer les liens religieux que chacun d'entre eux entretient, qu'il s'agisse de Nicéphore Soglo (1991-1996), de Mathieu Kérékou (1996, 2001) ou de Yayi Boni (2006 et 2011). La rumeur a attribué au premier d'avoir pactisé avec les chefs de culte vodun suite à sa guérison miraculeuse, la force de sa renaissance en Christ a transformé le second en sage et en Messie que l'on convoque à chaque nouvelle crise gouvernementale; quant au troisième, ses liens avec les Églises évangéliques sont publiquement assumés, y compris lors de ses voyages à l'étranger ${ }^{17}$.

Il apparaît donc, comme l'a souligné Mayrargue, que les conceptions de la politique sont partiellement orientées par l'appartenance religieuse, qui depuis le retour à la démocratie, s'avère plus diversifiée ${ }^{18}$. Ce sont moins les partis qui mobilisent les adhésions pour tel ou tel candidat que son appartenance à un réseau religieux transnational et puissant. Ce genre de réseau permet de mobiliser des fonds grâce à un double langage qui, d'un côté, stigmatise l'enrichissement rapide des non-chrétiens accusés de commerce avec les forces du mal, et de l'autre fait usage d'un discours moral où l'argent des Évangéliques est la preuve des bénédictions divines ${ }^{19}$.

Plus précisément encore, Souaré, en analysant les dernières élections présidentielles de 2011 où l'opposition s'était pour la première fois organisée en coalition pour tenter de battre le président en poste, attribue l'échec de l'opposition à son caractère trop marqué régionalement (le sud et le centre du pays) ainsi qu'à la manipulation par les autorités en poste de la LEPI (Liste électorale permanente informatisée), qui a oublié environ 700000 électeurs ${ }^{20}$, pour la plupart habitant des régions soutenant l'opposition. Souaré rappelle cependant que ce coup électoral a été facilité par le refus initial des membres de l'opposition de participer à la mise en place de cette liste informatisée.

Le rejet des partis s'explique aussi par le non-renouvellement des élites politiques. Tant Adrien Houngbédji, le candidat malheureux de trois élections consécutives, que les

\footnotetext{
${ }^{16}$ Cf. Issaka K. Souaré, « The 2011 presidential election in Benin : Explaining the success of one or two firsts », Journal of African Elections $10 \mathrm{n}^{\mathrm{o}} 2$ (2011) : 73-92.

${ }^{17}$ Voir par exemple sa visite à la mégaChurch de la région parisienne, Paris Centre chrétien : http://www.enseignemoi.com/page/video/visite-du-president-du-benin-yayi-boni-a-l-eglise-pcc-6552.html, mis en ligne le 29 janvier 2010.

${ }^{18}$ Cédrirc Mayrargue, «Identité religieuse et culture politique : les représentations de la démocratie des fidèles pentecôtistes au Bénin, Cadernos de estudos africanos 5-6 (2004) : 159-173.

${ }^{19}$ Meyer, «The power of money ».

${ }^{20}$ Souaré, « The 2011 presidential election in Benin », 85.
} 
jeunes loups, aujourd'hui vieillissants et plus connus pour les scandales qui ont entaché leur réputation au sein de différents gouvernements (par exemple, Séverin Adjovi, Bruno Amoussou, ou encore les fils Soglo, Léhandy et Ganiou), évoquent tout à la fois les maux d'une Afrique enferrée par le non-renouvellement de ses élites, la corruption, le régionalisme et le népotisme. Au-delà de ces maux qui ne sont pas propre à l'Afrique, on peut également constater une nette homologie entre les évolutions des champs religieux et politique depuis le tournant néolibéral: la concurrence y est dans les deux cas intense, on assiste à une individualisation et à une désinstitutionalisation des parcours dans le vaste marché théologicopolitique.

Ainsi il est possible d'observer une requalification en termes religieux des processus politiques $^{21}$, et la nouveauté réside principalement dans le fait que cette contamination du religieux au politique s'affiche ouvertement, comme en témoigne le slogan Dieu aime le Bénin lancé comme une litanie à chaque moment de tension lors des élections.

La perméabilité entre discours politique et symbolique religieuse a été très bien analysée par Claude Lefort; il la désigne par l'expression «théologico-politique » dont il rappelle la permanence dans le monde moderne, tout en soulignant qu'elle ne relève plus de l'ordre symbolique mais de l'imaginaire ${ }^{22}$. Quant au «réenchantement» du monde, il se caractérise par une désinstitutionalisation et une individualisation du rapport au sacré ${ }^{23}$. Dans une Afrique de plus en plus urbanisée, l'individualisme se manifeste au travers des choix religieux qui sont faits pour échapper au malheur et à l'infortune, mais également pour rompre avec certaines obligations lignagères ou claniques ${ }^{24}$.

\section{Les finances de Dieu et des scandales qui tombent à pic}

De l'« esprit de piratage » des réformistes chrétiens dès leur scission du catholicisme romain ${ }^{25}$ à l'éthique protestante de l'esprit capitaliste de Max Weber, de l'économie de la finance à

\footnotetext{
${ }^{21}$ Ces constatations ont aussi été faites par Maud Lasseur et Cédric Mayrargue, «Pluralisation religieuse, entre éclatement et concurrence », Politique africaine, 123 (2011) : 5-25. Sur l'instrumentalisation du religieux, en particulier des cultes vodun par l'ancien royaume du Danxomé, voir Robin Law, Ouidah : The Social History of a Western African Slaving "Port", 1727-1892 (Athens: Ohio University Press-Oxford: James Currey, 2004), Edna G. Bay, Wiwes of the Leopard: Gender, Politics and Culture in the Kingdom of Dahomey (Charlottesville : University Press of Virginia, 1998), et E. Kadya Tall, « De la démocratie et des cultes vodun », Cahiers d'études africaines 137 (1995) : 195-208.

${ }^{22}$ Claude Lefort, « Permanence du théologico-politique », dans Essais sur le politique (Paris : Le Seuil « Points », 1986 [1981]) : 275-329.

${ }^{23}$ Patrick Michel, « Postface », Critique internationale $22 \mathrm{n}^{\circ} 1$ (2004) : 139-143.

${ }^{24}$ Voir Alain Marie, « Du sujet communautaire au sujet individuel : une lecture anthropologique de la réalité africaine contemporaine », dans L'Afrique des individus, dir. Alain Marie (Paris : Karthala, 1997) : 53-110.

${ }^{25}$ Voir l'article d'Olivier Abel dans lequel il écrit que «la piraterie appartient à une histoire politique et idéologique de longue durée qui nous ramène à la naissance du protestantisme ». En effet, la Réforme a permis
} 
l'enrichissement en liquidités - or et biens précieux au premiers temps de la conquête du Nouveau Monde et aujourd'hui en titrisation -, l'enrichissement au nom de Dieu semble être une constante.

Depuis le retour à la démocratie au Bénin, c'est le modèle du capitalisme néolibéral qui semble avoir conquis les électeurs béninois. En effet, de Nicéphore Soglo au sortir de la Conférence nationale (1991), à l'élection de Yayi Boni en 2006, le peuple béninois a toujours élu quelqu'un censé avoir les compétences qui permettraient au pays de sortir de sa stagnation économique, en partie due au plan d'ajustement structurel et à l'absence de ressources naturelles à fort rendement dans le pays. Les deux élus avaient été adoubés parce qu'ils avaient l'un et l'autre acquis une expérience dans des institutions financières internationales. Le premier avait été le représentant du Bénin auprès de la Banque mondiale, quant au second, il avait été directeur de la Banque ouest africaine pour le développement (BOAD) avant de briguer la charge présidentielle.

Nicéphore Soglo n'a fait qu'un mandat et a échoué à sa propre succession. On ne s'attardera pas sur les raisons qui ont causé son échec à sa seconde candidature à la magistrature suprême, si ce n'est pour rappeler que son soutien à la cause vodun lui a fait sans aucun doute perdre une grande partie de l'électorat chrétien, plus particulièrement catholique. En effet, la patrimonalisation des vodun et du royaume d'Abomey, ainsi que l'affaire du portrait de Béhanzin en filigrane sur les passeports nationaux en 1995 a de surcroît été perçue comme une tactique d'empowerment régionaliste pour l'électorat des départements du nord du pays ${ }^{26}$.

Dans le cas de Yayi Boni, il est important de noter sa réélection. En effet, contrairement à Soglo, son régionalisme ne l'a pas empêché de gagner les élections présidentielles de 2011: la manipulation de la liste électorale LEPI et la coalition de ses opposants, tous issus de la région méridionale du pays, ont grandement favorisé sa reconduite à la tête du pays. Il faut cependant s'interroger sur sa longévité, d'autant que, très vite, les scandales se sont succédés et que le chef de l'État a manifesté une propension à entretenir un culte de la personnalité et à brider les contre-pouvoirs que sont les médias et l'opinion publique. Ces scandales sur fond de corruption et de pratiques liberticides touchent à la fois les champs politique et religieux. Il suffit de retenir les deux cas suivants.

que les pilleurs soient à leur tour pillés. Les pilleurs sont ces conquistadores et leur flotte qui, parce qu'ils faisaient partie du vaste empire ibérique et catholique, pouvaient razzier or et biens précieux et les rapporter des terres du Nouveau monde, voir Olivier Abel, «Introduction : L'océan, le puritain, le pirate », Esprit 7 (2009) : 104.

${ }^{26}$ Tilo Grätz, «La rébellion de Kaba (1916-1917) dans l'imaginaire politique au Bénin », Cahiers d'études africaines 160 (2000) : 675-676. 


\section{Le scandale de ICC Services}

Un an après l'arrivée au pouvoir de Yayi Boni, la société Investment Consultancy and Computering Services (ICC Services) obtient son inscription au registre de la Chambre de commerce et d'industrie du Bénin afin de pouvoir lancer ses activités de dépôts de fonds, tontine et épargne. Les responsables de cette société sont des Chrétiens célestes et, bien que le chef de l'État appartienne à une autre obédience religieuse - il est évangélique ${ }^{27}$-, il soutient leur action. Au début de l'année 2008 et surtout en 2009, la masse d'argent que brassait cette société a commencé à attirer l'attention d'instances financières telle que la Banque centrale des États de l'Afrique (BCEAO) mais également celle des banques de micro-crédit, ce qui a conduit cette entreprise à devoir soumettre un dossier de façon à obtenir un agrément officiel. Selon certaines rumeurs, le Palais de la Marina a tout fait pour bloquer cet agrément afin que les noms des personnes proches du pouvoir et impliquées dans cette société d'investissement fonctionnant sur le principe éprouvé de la fraude pyramidale ${ }^{28}$ ne soit pas dévoilés.

$\mathrm{Au}$ vu de ce genre de scandale, et au-delà de la politique du ventre si souvent dénoncée en Afrique, on peut se demander s'il n'y a pas plutôt eu un renversement des perspectives, à savoir qu'il s'agirait ici moins de clientélisme que du pouvoir qu'a une certaine élite à voler son peuple. La fiction de la politique du ventre oblitère le fait que, dans beaucoup de pays d'Afrique, ce sont les puissants qui affament le peuple et les appauvrissent en leur faisant miroiter des gains immédiats et invraisemblables. L'impératif de moralisation de la vie publique brandi par la plupart des mouvements réformateurs religieux, qui semble faire écho aux exigences des organismes internationaux, a pour corollaire un dogme paradoxal particulièrement frappant, celui de faire croire aux fidèles de ces mouvements que la puissance de l'argent serait la preuve d'une appartenance au peuple des élus de Dieu. Recevoir les bénédictions de Dieu en numéraire permet de redistribuer ses grâces autour de soi. Dans tous les cas, on n'est jamais dans la redistribution mais dans la dépense.

\footnotetext{
${ }^{27}$ Il a un temps été membre de l'Église Foursquare comme Laurent Gbagbo, mais aujourd'hui, il ferait partie de l'Assemblée de Dieu, voir Fiacre Vidjingnignou, «Bénin : les béni-oui-oui de Boni Yayi », Jeune Afrique du 6 février 2014: http://www.jeuneafrique.com/Article/JA2768p024.xml9/b-nin-boni-yayi-pentecotisme-religionglises-vang-liques-b-nin-les-b-ni-oui-oui-de-boni-yayi.html.

${ }^{28}$ Cette fraude fonctionne de la façon suivante : chaque membre de la société doit s'acquitter d'une certaine somme pour y entrer, et son recruteur reçoit une part de ce droit d'entrée, une autre part étant répartie dans la chaîne des recruteurs successifs. La forme et la part qui reviennent aux membres déjà dans le réseau (recruteur, recruteur du recruteur, etc.) dépend de la pyramide. Le plus souvent la pyramide prend grand soin de dissimuler au recruté que ce droit d'entrée bénéficie au recruteur.
} 


\section{Les accusations de corruption du clergé catholique}

Pour comprendre l'impression de délitement social qui a submergé la population lorsque différents scandales ont touché le clergé catholique, obligeant le Vatican à exiger la démission des coupables avant la visite du Pape en 2011, il convient de rappeler le rôle central qu'a eu l'Église catholique dans la mise en place de la Conférence nationale qui allait mettre fin à dixsept ans de régime autoritaire au Bénin.

«Le Vatican, il est vrai, a toujours choyé ce minuscule Bénin, dont Jean-Paul II foula le sol à deux reprises, et qui envoya à Rome d'éminents cardinaux, tel Bernardin Gantin, pilier de la Curie tenu pour "papabile" en 1978. »"

Pourtant, dès son premier mandat des scandales touchent régulièrement le gouvernement de Yayi Boni : il y a une rotation effrénée des ministres et plusieurs esclandres éclaboussent également d'autres institutions. En particulier l'Église catholique qui est confrontée à des accusations de franc-maçonnerie, et/ou d'avoir participé à un culte néovodun pour l'Évêque de Porto Novo devenu archevêque de Cotonou avant sa démission, et de mœurs dégradés pour l'Évêque de Parakou, une accusation, provoquant la démission forcée des deux évêques avant la visite du pape Benoît XVI en novembre 2011. À l'instar des Églises évangéliques, les cultes néovodun et la franc-maçonnerie prêchent la multiplication des richesses, mais contrairement aux Évangéliques, ce n'est pas la puissance de Dieu qui est invoquée pour recevoir ses grâces, mais celles d'entités du monde supranaturel que les adeptes sollicitent en se livrant à des rituels sacrificiels, que leurs détracteurs soupçonnent souvent d'être anthropophagiques.

Les accusations portées contre les plus hauts membres du clergé catholique ont ébranlé la foi de certains fidèles qui découvraient brutalement la faillibilité des hommes d'Église qui avait été porteurs des idéaux de démocratie et de justice lors de la Conférence nationale. La corruption des mœurs et la financiarisation des rapports sociaux touchaient désormais ceux qui avaient fait vœux de chasteté et de pauvreté pour servir l'Église. Si la question du célibat n'avait jamais véritablement posé problème dans un pays où la virilité se mesure au nombre d'enfants procréés dans et hors mariage, il devenait soudainement nécessaire de dénoncer les mondanités et la promiscuité que certains membres du clergé entretenaient avec des jeunes gens ; c'est ainsi que les mœurs d'un jeune prêtre, très élégant, amateur de vins fins et de jeune compagnie sont alors devenus la cible des détracteurs. C'est ce climat de dénonciation

\footnotetext{
${ }^{29}$ Vincent Hugueux, « Bénin : le culte qui défie l'Église », L'Express, 14 mars 2013 : http://www.lexpress.fr/actualite/monde/afrique/benin-le-culte-qui-defie-l-eglise_1239516.html.
} 
des turpitudes du clergé qui a été le ferment qui a permis l'éclosion d'un mouvement de dissidence.

L'ethos des différentes formations religieuses touchant aux finances de Dieu témoigne d'un même universalisme reposant sur une fiction, la même que nous avons également rencontrée sur d'autres terrains, au Brésil par exemple. Il s'agit de cet ethos que Bolivar Etcheverría a qualifié de baroque parce que, dans les sociétés post-capitalistes et néo-libérales ayant été victimes de l'emprise coloniale européenne, cet ethos met en avant la valeur d'usage plutôt que la valeur marchande ${ }^{30}$. Cette fiction de l'enrichissement rapide pour pouvoir dépenser nous semble en effet participer de cet ethos où la financiarisation est moins dans le cumul que dans l'ostentation.

\section{L'émergence d'une nouvelle Église : l'Église de Banamè}

Depuis fin 2009, l'Église qui fait le plus parler d'elle au Bénin est l'Église de Banamè - en référence à la localité où elle est en train de construire son siège, dans le département du Zou, à quelques kilomètres de l'ancienne capitale du royaume d'Abomey, sur la colline de Sovidji. Cette commune bénéficie d'une renommée locale comme haut lieu de sorcellerie, et le fait que le prêtre et les deux petites filles s’y soient réfugiés sans encombre après que la hiérarchie de l'Église catholique romaine l'a radié, ajoute à la puissance du lieu en train d'être transformé en Vatican de la nouvelle Église. Voici comment la formation de la Trinité y est contée :

«Le père Mathias Vigan venait d'être officiellement nommé comme un exorciste du diocèse d'Abomey, en octobre 2008. Quelques faits isolés ont accueilli ce ministère à Banamè : délivrance d'un esprit malin, guérison physique par suite de prière de délivrance... Mais il devait y avoir plus, à partir du 11 novembre 2008, avec l'arrivée de la fille Nicole Soglo ${ }^{31}$. Le démon qui révèle son identité, ses astuces de nuisance, et bien des choses de l'invisible restées toujours cachées aux hommes victimes. L'extinction du démon et de la sorcellerie, qui est devenue une réalité permanente. Et depuis l'arrivée le 20 janvier 2009 de Parfaite qui voit sans faille dans la vie et dans la conscience de chacun... L'une et l'autre sont arrivées, elles aussi, pour être délivrées de maladie: Nicole avec ses problèmes d'yeux, Parfaite avec ses obsessions démoniaques paralysantes. $»^{32}$

Le décor est planté, les naissances des divinités font suite à la rencontre avec le père Mathias Vigan. Nicole, la première, est née le 11 novembre 2008. Recouvrant la vue, elle devient la femme qui écrase la tête du serpent, comme annoncé dans les Écritures. Transfigurée en Sainte Vierge, elle sert également de support aux démons qui confessent la vérité et

\footnotetext{
${ }^{30}$ Voir Bolívar Echeverría, « La clave barroca de la Americana latina » (Conférence donnée à l’Institut latinoaméricain de l'Université libre de Berlin en novembre 2002) : http://www.bolivare.unam.mx/ et E. Kadya Tall, Le candomblé de Bahia, miroir baroque des mélancolies postcoloniales (Paris : Éd. du Cerf, 2012).

${ }^{31}$ La jeune fille souffrant de troubles de la vue qui deviendra le réceptacle de la Sainte Vierge.

${ }^{32}$ Évangiles de l'Église de Banamè, s.l. n. d., Tome 1 : La Vierge Marie notre mère doit écraser l'antique serpent, l'heure a sonné pour l'humanité à Banamè, 3.
} 
s'éteignent. La rencontre de celle qui allait successivement devenir Parfaite puis Dieu/Daagbo, c'est-à-dire la mise en présence réelle de Dieu sur terre, date, selon le premier Évangile de l'Église, du 20 janvier 2009. Comme dans la plupart des récits de conversion, sa naissance est extraordinaire. Selon la version officielle, elle n'est pas Vicencia, la fille de Raymond Chanvoukini et de Victorine Hounwèdo, mais une orpheline, ramassée lorsqu'elle était nourrisson par un berger peul dans la brousse de Bembèkèrè. C'est la Sainte Vierge qui lui aurait dévoilé la vérité de son origine sur la colline de Sovidji.

« Parfaite n'est pas un être humain.

[...] Car Parfaite est sans généalogie, elle n'est pas née de chair et de sang.

Parfaite délivrée de ses obsessions se transforme en l'Esprit Saint. ${ }^{33}$

Les deux petites filles transfigurées «calcinent ${ }^{34}$ désormais la sorcellerie des humains qui viennent se mettre sous leur protection. En effet, dans cette Église, «le Diable n'est pas seulement chassé, mais calciné ». Enfin, le père Mathias est le grand ordonnateur de la découverte des dons médiumniques des deux jeunes-filles. Nicole recevant la mère de Dieu et Parfaite, Dieu, l'Esprit Saint. «Et voilà qu'aujourd'hui Nicole, Mathias et Parfaite sont devenus trois outils d'un même instrument de salut pour tous, salut dont l'initiative appartient à notre chère maman, la Vierge Marie. $»^{35}$

Au Bénin, même si ce n'est pas un hasard que l'Église universelle du Règne de Dieu soit absente, la manière dont l'Église de Banamè use et abuse des provocations pour faire parler d'elle n'est pas sans rappeler les actions de l'EURD (Église universelle du Royaume de Dieu) au Brésil, dont la plus marquante demeure l'entretien télévisé d'un pasteur qui bousculait la statue de la Sainte patronne du Brésil ${ }^{36}$, le 12 octobre 1995, jour anniversaire de la découverte de l'Amérique par Christophe Colomb.

En reprenant des dates de la patrimonialisation des cultes locaux (10 janvier, jour férié dédié aux cultes vodun depuis 1996), l'Assumption de la Vierge Marie (15 août), ou encore profitant de la date anniversaire du séjour du Pape Benoît XVI pour célébrer la naissance de Parfaite/Daagbo/Dieu le 17 novembre 2012, et faire en sorte que le lendemain, Daagbo/Dieu/Parfaite proclame le prêtre diocésain Mathias Vigan pape Christophe XVIII, non seulement la Trinité de l'Église de Banamè manipule la mémoire des cultes précoloniaux

\footnotetext{
${ }^{33}$ Ibid., 21, 22.

${ }^{34}$ Calciner, qui signifie soumettre à une très forte température pour obtenir une combustion totale, est utilsé dans le vocabulaire de ce mouvement pour signifier son efficacité à faire disparaître irrémédiablement le pouvoir sorcier des individus touchés par ce phénomène, qu'il soit volontaire ou involontaire...

${ }^{35}$ Ibid., 3.

${ }^{36}$ Nossa Senhora da Conceição Aparecida, figuration noire de la Sainte Vierge qui est célébrée le jour de la découverte de l'Amérique par Christophe Colomb depuis que le pape Jean-Paul II a consacré la basilique qui l'abrite depuis 1980 .
} 
et leur patrimonialisation, et les profane, mais elle se joue également des moments importants de l'Église catholique romaine en choisissant la date de la visite du Pape pour introniser son Dieu et le jour suivant pour introniser son propre Pape.

En s'affirmant en opposition aux cultes vodun et à l'Église catholique romaine officielle, l'Église de Banamè procède d'autant plus comme les Églises évangéliques que son angle d'attaque est la calcination de la sorcellerie et des démons en tout genre. Parmi les innovations du mouvement qui le rapprochent des Églises protestantes, il faut noter le mariage des prêtres avec néanmoins le maintien des ordres célibataires masculins et féminins.

\section{La pentecôtisation des religions}

Le Renouveau charismatique, courant de l'Église catholique né aux États-Unis à la fin des années 1960, est souvent qualifié de «pentecôtisme catholique ${ }^{37}$ dans la mesure où ce mouvement se rattache à la tradition des « réveils », c'est-à-dire que l'accent est mis sur l'expérience spirituelle de conversion, sur l'Esprit saint et qu'une grande attention est portée aux charismes, notamment à celui de guérison. En Afrique subsaharienne, la pentecôtisation touche également les mouvements islamiques réformateur ${ }^{38}$ qui puisent dans la désinstitutionalisation du charisme l'expérience personnelle de la conversion et dans l'usage des nouvelles technologies médiatiques les outils pour s'affranchir d'une culture islamique ancrée dans la coutume et ses hiérarchies, particulièrement manifeste dans l'islam soufi confrérique.

Parmi la dizaine d'entretiens que nous avons menés en août 2014 avec des fidèles et des membres de la hiérarchie de la communauté ecclésiastique de Banamè, la majorité d'entre eux étaient auparavant des fidèles de l’Église catholique. Ainsi, deux anciennes religieuses aujourd'hui cardinales, un ancien séminariste dominicain devenu abbé qui, adolescent, a été témoin des fréquentations franc-maçonne et sorcière de l'évêque de Porto Novo, un autre devenu cardinal après avoir été banquier, occupe les fonctions de communiquant à l'international, comme le cardinal Simon, ancien patron de presse et toujours directeur d'une radio privée dans le département du Zou. Un évêque et un prêtre, tous deux récemment promus dans le district d'Abomey, étaient des enseignants, l'un instituteur, l'autre professeur

\footnotetext{
${ }^{37}$ Voir Martine Cohen, « Vers de nouveaux rapports avec l'institution ecclésiastique : l'exemple du Renouveau charismatique en France », Archives des sciences sociales des religions 62 n$^{\circ} 1$ (1986) : 61-79.

${ }^{38}$ Voir Michel, « Postface », et Cédric Mayrargue, « Pluralisation et compétition religieuse en Afrique subsaharienne : pour une étude comparée des logiques sociales et politiques du christianisme et de l'islam », Revue internationale de politique comparée $16 \mathrm{n}^{\circ} 1$ (2009) : 83-98.
} 
de langue au lycée. Parmi notre échantillon, des membres de la nouvelle Église ont accompagné le Renouveau charismatique.

La Sainte Colline de Sovidji est devenue le rendez-vous hebdomadaire de personnes en quête de solution à leurs problèmes médicaux ou professionnels. Depuis que les rassemblements de l'Église ont été interdits en raison des violences qui accompagnaient les messes organisées au stade de l'amitié ou dans des communes avoisinant Cotonou, la Sainte Trinité a pour objectif de construire sa cité papale sur le territoire de Banamè. Chaque date importante du calendrier de l'Église (Pentecôte, Assumption et le jour anniversaire de Daagbo-Parfaite-Dieu) est l'occasion de consacrer des fidèles à des charges sacramentelles (cardinaux, évêques, ordre de frères et de religieuses, abbés, etc.) pour constituer une cité-État semblable au Vatican. C'est d'ailleurs sur la terminologie des charges et de l'identité nominale utilisée par le mouvement que l'Église catholique attaque régulièrement le prêtre excommunié et ses fidèles. Elle lui dénie le droit de s'appeler Église catholique universelle romaine, et s'appuie sur son autorité pour enjoindre les autorités juridiques à repousser sine die la reconnaissance du mouvement comme association régie par la loi de 1901. Ce à quoi Dieu-Daagbo-Parfaite réagit violemment dans ses diatribes contre 1'Église catholique, en arguant de la laïcité de l'État béninois qu'a instauré la nouvelle constitution de 1991.

La cosmologie de Banamè met l'accent à la fois sur des figures de la culture vodun, sur celles de la culture du Christianisme Céleste (les Saints combattants le démon), et se crée ses propres figures issues du christianisme comme saint Étienne premier martyr. En ce qui concerne la figure de la Sainte Vierge, elle peut paraître très œcuménique puisqu'elle est appréciée autant des catholiques, des chrétiens céleste et des musulmans. Sans oublier que la Vierge Marie est la figure inversée de Mami Wata, la déesse des eaux douces et salées ${ }^{39}$.

L'importance de la Vierge dans la cosmologie de Banamè, où rappelons-le, la Trinité est formée par Dieu, le Pape et la Sainte Vierge, est manifeste durant la fête mariale du 15 août. Pour rendre compte de l'expérience vécue d'une fin de pèlerinage dans un pays peuplé de 10 millions d'habitants, où la sensation d'oppression du fait de la foule ne se rencontre qu'à l'issue d'un match de football ou lors du jour du marché de Dantokpa ${ }^{40}$, arriver à Banamè a été d'autant plus une expérience rare qu'à la même époque, l'épidémie d'Ebola

\footnotetext{
${ }^{39}$ Mami Wata, littéralement « mère des eaux », est une figure de la sexualité féminine que l'on rencontre dans toute l'Afrique de l'Ouest, mais sa puissance est particulièrement vivace dans les pays fortement influencés par le christianisme importé par les colons. Voir Joseph Tonda, La guérison divine en Afrique centrale (Paris : Karthala, 2002).

${ }^{40}$ Dantokpa est le grand marché de Cotonou et également le plus grand marché à ciel ouvert d'Afrique de l'Ouest.
} 
explosait en Afrique de l'Ouest et qu'un cas avait été déclaré au Nigeria voisin. Voici les notes de terrain rédigées après cette visite :

Nous arrivons à Banamè le dimanche 17 août 2014, vers 13 heures. Chaleur écrasante. Contrairement au département des Collines, il ne semble pas qu'il ait plu ces derniers jours. L'accès à la Colline est interdit aux véhicules en raison du déjà très grand nombre de véhicules [...]. Çà et là des pancartes indiquant Dagbovi [i.e. enfant du/de la Daagbo/Dieu] propose des douches et WC à 50 et $100 \mathrm{CFA}$. Des marchandes de nourriture et boissons sur le flanc de la colline [...] Poussière, odeur d'urine et de pourriture prennent à la gorge [...]. La couleur de Daagbo, alias Parfaite, est le rouge. Je vois également des personnes avec un fichu bleu sur lequel je note Notre Dame de Lourdes 10 janvier ${ }^{41}$. Sur le fichu rouge, est inscrit en lettre majuscule FEU et mon assistant me confirme que les gens parlent de $\mathrm{Zo}^{42}$, qui est également, du moins à Ouidah, un vodun. [...]Des familles entières, et surtout beaucoup de jeunes, avec ou sans leur parents. Des personnes me photographient avec leur portable en criant yovo yovo, signe que ma présence vient légitimer ce rassemblement.

Impression générale, beaucoup de jeunes, et pas seulement des femmes accompagnées de jeunes enfants comme c'est bien souvent le cas dans les cultes évangélistes. J'entends le terme Gbigbowiwe (respiration blanche célébrée d'abord par les chrétiens céleste) chanté à plusieurs reprises Sur un parking, une dizaine de 4/4 flambant neufs.

L'évocation du feu Zo qui calcine la sorcellerie est un détournement de cet élément considéré comme un vodun. Du feu vengeur d'un vodun malmené, Zo devient le feu expiatoire qui permet de se libérer de ses démons et sorciers. Cette évocation est redoublée par la mention sur les fichus des fidèles du 10 janvier, jour férié dédié aux vodun, comme pour en inverser la signification. Il est intéressant de noter que les sorciers s'auto-dénoncent en foulant le sol de la Sainte colline, ce qui n'est pas sans poser des problèmes. En effet, à l'instar de ce qui se passe dans les Églises congolaises ${ }^{43}$, de plus en plus de jeunes enfants s'auto-dénoncent, ce qui provoque leur abandon sur place par leurs parents. Une des cardinales interrogées nous a confié qu'à Abomey un orphelinat comptant déjà deux cents enfants abandonnés les prenait en charge. Avec l'aide du premier martyr de l'Église, saint Étienne, il s'agit, en se dénonçant, de libérer chacun de ses démons pour permettre à Dieu de couvrir les fidèles de ses libéralités. La richesse des parements des membres du nouveau clergé, sans compter le luxe de leur voiture tout terrain, est là pour démontrer l'efficacité du mouvement.

À l'instar des mouvements néo-pentecôtistes, la communauté ecclésiastique de Banamè joue de l'imaginaire sorcellaire pour attirer à soi les nombreux mécontents du pays. Tous les fidèles ou membres du nouveau clergé que nous avons interrogés expliquent leur entrée dans ce nouveau mouvement en raison d'attaques en sorcellerie les rendant malades et/ou freinant leur ascension sociale. Mais si tous accusent la sorcellerie comme étant à

\footnotetext{
${ }^{41}$ Le 10 janvier est au niveau national, le jour de la célébration du vodun.

${ }^{42}$ Zo littéralement le feu est également un vodun clanique à Ouidah, auquel appartient la famille d'Angélique Kidjo. Cette référence à un vodun clanique de Ouidah s'explique du fait que le premier festival des cultures vodun, qui a rassemblé une foule venue du monde entier, s'est déroulé à Ouidah en février 1993 (Tall, « De la démocratie et des cultes vodun »).

${ }^{43}$ Pour l'histoire de Magali, une enfant-sorcier, voir Geneviève N'Koussou, « Magali », Cahiers d'études africaines 189-190 (2008) : 283-296, et Patrice Yengo, " Le monde à l'envers: enfance et kindoki ou les ruses de la raison sorcière dans le basin du Congo », Cahiers d'études africaines 189-190 (2008) : 297-323
} 
l'origine de tous leurs maux avant d'avoir rencontré le vrai chemin de Dieu pour s'en protéger, chacun se garde bien d'avouer sa part de responsabilité dans l'agression des sorciers qui les troublent et/ou leur barrent le chemin du succès et de la prospérité. Cet imaginaire de la sorcellerie ne rompt en aucune façon avec une vision persécutive du malheur et de l'infortune $^{44}$ : le coupable est toujours l'autre, fut-il la chair de sa propre chair.

Il est encore trop tôt pour prédire si ce mouvement de contestation de l'Église catholique romaine va perdurer et se développer. L'enrichissement rapide des membres du clergé fait soupçonner une escroquerie à la vente pyramidale ${ }^{4546}$ et c'est sans doute la raison pour laquelle, Dieu, son Pape et la Sainte Vierge accélèrent les promotions et la création de différents ordres religieux afin de couvrir une étendue de plus en plus grande du territoire national, tout en envoyant des émissaires dans certains autres pays d'Afrique (Cameroun et Côte d'Ivoire, notamment). La Sainte Trinité fait aussi des voyages réguliers en Chine pour y acheter les différents matériaux de construction de la nouvelle cité-État sur la colline de Sovidji.

Les échéances électorales présidentielles de 2016 vont sans nul doute changer le paysage religieux béninois dans la mesure où Yayi Boni, selon la Constitution, ne pourra plus se présenter pour un troisième mandat. La réussite de cette nouvelle Église, pas encore reconnue légalement, dépendra de l'habileté de son directoire à renouveler son discours contestaire des nouvelles autorités en poste.

\section{Bibliographie}

Abel, Olivier, «Introduction : L'océan, le puritain, le pirate », Esprit 7 (2009) : 104-110.

Augé, Marc et Claudine Herzlich. Le sens du mal, histoire, sociologie de la maladie. Paris : Éditions des Archives contemporaines, 1983

Banégas, Richard. La démocratie à pas de caméléon : transition et imaginaires politiques au Bénin. Paris : Karthala, 2003.

Bay, Edna G. Wiwes of the Leopard: Gender, Politics and Culture in the Kingdom of Dahomey. Charlottesville : University Press of Virginia, 1998.

\footnotetext{
${ }^{44}$ Sur ce sujet, pour le Sénégal, voir Andras Zempléni, Causes, origines et agents de la maladie chez les peuples sans écriture (Paris : Société d'ethnographie de Paris, 1985) et sur l'interprétation de la maladie et de l'infortune en l'Afrique et en France, voir Marc Augé et Claudine Herzlich, dir., Le sens du mal, histoire, sociologie de la maladie (Paris : Éditions des Archives contemporaines, 1983).

45 Sur ce genre de fraude, voir « Comprendre l'affaire Madoff », Le Monde, 19 déembre 2008 : http://www.lemonde.fr/economie/article/2008/12/19/comprendre-1-affaire-madoff_1133354_3234.html.
} 
Bayart, Jean-François. L'État en Afrique : la politique du ventre. Paris : Fayard « L'espace du politique », 1989.

Chatwin, Bruce. The Viceroy of Ouidah. London : Jonathan Cape, 1980.

Ciarcia, Gaetano. «Réthoriques et pratiques de l'inculturation : une généalogie "morale" des mémoires de l'esclavage au Bénin », Gradhiva 8 (2008) : 28-47.

Cohen, Martine. « Vers de nouveaux rapports avec l'institution ecclésiastique : l'exemple du Renouveau charismatique en France ", Archives des sciences sociales des religions 62 $\mathrm{n}^{\mathrm{o}} 1$ (1986) : 61-79.

Comaroff, Jean, et John Comaroff, "Occult Economies and the Violence of Abstraction : Notes from the South African Postcolony", American Ethnologist 26 n 2 (1999) : 279301 .

- dir. Modernity and its Malcontents: Ritual and Power in Postcolonial Africa. Chicago: University of Chicago Press. 1993.

Corten, André, Dozon, Jean-Pierre et Oro, Ari Pedro. Les nouveaux conquérants de la foi : l'Église universelle du royaume de Dieu, Brésil. Paris : Karthala, 2003.

Echeverría, Bolívar. "La clave barroca de la Americana latina », Conférence donnée à l'Institut latino-américain de l'Université libre de Berlin en novembre 2002: http://www.bolivare.unam.mx/

Évangiles de l'Église de Banamè. La Vierge Marie notre mère doit écraser l'antique serpent, l'heure a sonné pour l'humanité à Banamè. S.l.n.d., Tome I, 43 p.

- La Vierge Marie notre mère doit écraser l'antique serpent, Le Dieu et Père de Jésus Christ qui a pris chair à Banamè interpelle. S.1.n.d., Tome 2, 94 p.

- L'heure a sonné pour l'humanité. C'est la fin du règne de Belzébul et de ses suppôts sur le monde entier. S.1.n.d., Tome 3, 76 p.

- Banamè : Dieu dans la chair. Cinq ans après, où en sont les hommes ? S.1.n.d., Tome 4, $130 \mathrm{p}$.

Geschiere, Peter. Sorcellerie et politique en Afrique : la viande des autres. Paris : Karthala. 1995.

- The Modernity of Witchcraft: Politics and the Occult in Postcolonial Africa. Charlotsville : University of Virginia Press, 2003 [1997].

- «Witchcraft and modernity : Perspectives from Africa and beyond », dans Sorcery in the Black Atlantic, dir. Luis Nicolau Parès et Roger Sansi, 233-258. Chicago-London : The Chicago University Press, 2011.

Grätz, Tilo. «La rébellion de Kaba (1916-1917) dans l'imaginaire politique au Bénin », Cahiers d'études africaines 160 (2000) : 675-704.

Henry, Christine. La force des anges : rites, hiérarchie et divination dans le Christianisme Céleste (Bénin). Turnhout, Brepols: Bibliothèque de l'École des hautes études, Sciences religieuses, vol. 135 (2008).

Hugueux, Vincent, « Bénin : le culte qui défie l'Église », L'Express, 14 mars 2013 : http://www.lexpress.fr/actualite/monde/afrique/benin-le-culte-qui-defie-1eglise_1239516.html 
Lasseur, Maud \& Mayrargue, Cédric. «Pluralisation religieuse, entre éclatement et concurrence », Politique africaine, 123 (2011) : 5-25.

Law Robin. Ouidah: The Social History of a Western African Slaving "Port", 1727-1892. Athens : Ohio University Press-Oxford : James Currey, 2004.

Lefort, Claude. «Permanence du théologico-politique», dans Essais sur le politique. Paris : Le Seuil « Points », 1986 [1981] : 275-329.

Marie, Alain, « Du sujet communautaire au sujet individuel : une lecture anthropologique de la réalité africaine contemporaine », dans L'Afrique des individus, dir. Alain Marie, 53110. Paris : Karthala, 1997.

Marin, Louis. « Baroque, Classique : Versailles ou l'architecture du Prince », dans Politiques de la représentation, 247 -261. Paris : Kimé, 2005.

Maupoil, Bernard. La géomancie à l'ancienne Côte des Esclaves. Paris : Institut d'ethnologie, (1981 [1943]).

Mayrargue, Cédric, «Les élites politiques béninoises au temps du Renouveau démocratique: entre continuité et transformation ", dans Le non renouvellement des élites en Afrique subsaharienne, dir. Jean-Pascal Daloz et al., 33-56. Bordeaux: Centre d'étude d'Afrique noire, 1999.

— «Identité religieuse et culture politique : les représentations de la démocratie des fidèles pentecôtistes au Bénin, Cadernos de estudos africanos 5-6 (2004) : 159-173.

- «Pluralisation et compétition religieuse en Afrique subsaharienne : pour une étude comparée des logiques sociales et politiques du christianisme et de l'islam ", Revue internationale de politique comparée, $16 \mathrm{n}^{\circ} 1$ (2009) : 83-98.

Mboukou, Serge. Messianisme et modernité : dona Béatrice, Kimpa Vita et le mouvement des Antoniens. Paris : L'Harmattan, 2010.

Meyer, Birgit. " "If you are a devil, you are a witch and, if you are a witch you are a devil" : The integration of "Pagan" ideas into the Conceptual universe of Ewe Christians in Southern Ghana », Journal of Religion in Africa, $22 \mathrm{n}^{\circ} 2$ (1992) : 98-131.

— « The power of money : politics, occult forces, and Pentecostalism in Ghana », African Studies Review 41 n $^{\circ} 3$ (1998) : 15-37.

Michel, Patrick. « Postface », Critique internationale 22 n 1 (2004) : 139-143.

N'Koussou, Geneviève, « Magali », Cahiers d'études africaines 189-190 (2008) : 283-296.

Souaré, Issaka K. «The 2011 presidential election in Benin : Explaining the success of one or two firsts », Journal of African Elections $10 \mathrm{n}^{\mathrm{o}} 2$ (2011) : 73-92.

Strandsbjerg, Camilla. « Continuité et rupture dans les représentations du pouvoir politique au Bénin entre 1972-2001 : le président Kérékou, du militaire-marxiste au démocrate pasteur », Cahiers d'études africaines 177 (2005) : http://etudesafricaines.revues.org/4903.

— «Les sens du pouvoir. Des forces" occultes" à la grâce divine: religion et transformations politiques dans le Bénin contemporain », thèse de doctorat, École des haites études en sciences sociales, Paris, 2008. [À paraître : Religion et transformations politiques au Bénin : les spectres du pouvoir, Karthala, 2015].

Sundkler, Bengt. Bantu Prophets in South Africa. Cambridge : James Clarke, 2004 [1948]. 
Tall, E. Kadya. «De la démocratie et des cultes vodun », Cahiers d'études africaines 137 (1995) : 195-208.

— «Stratégies locales et relations internationales des chefs de culte au Sud-Bénin », dans Entreprises religieuses transnationales en Afrique de l'Ouest, dir. Laurent Fourchard, André Mary et René Otayek, 267-284. Paris : Karthala, 2005.

- Le candomblé de Bahia, miroir baroque des mélancolies postcoloniales. Paris : Éd. du Cerf, 2012.

Terray, Emmanuel. « Afrique ambiguë : le climatiseur et la véranda », dans Afrique plurielle, Afrique actuelle. Hommage à Georges Balandier. Paris : Karthal, 1986 : 37-44.

Tonda, Joseph. La guérison divine en Afrique centrale. Paris : Karthala, 2002.

Yengo, Patrice. "Le monde à l'envers: enfance et kindoki ou les ruses de la raison sorcière dans le basin du Congo », Cahiers d'études africaines 189-190 (2008) : 297-323.

Zempléni, András. Causes, origines et agents de la maladie chez les peuples sans écriture. Paris : Société d'ethnographie de Paris, 1985

\section{Résumé}

Cette étude s'intéresse à un mouvement de contestation de l'Église catholique romaine fondé par un prêtre exorciste et deux jeunes femmes médiums dont il a révélé la nature divine en 2009. Ce mouvement est né dans un contexte où après 20 ans de retour à la démocratie, la population béninoise est confrontée au non renouvellement des élites politiques du pays, à la corruption et au régionalisme. La plupart des fidèles de ce mouvement encore trop jeune et pas encore légalisé pour être considéré comme une Église nouvelle, appartient en majorité à une jeunesse urbaine en attente et en manque de promotion sociale.

Mots-clefs:.

\section{Abstract}

This paper is concerned with a movement that challenges the Catholic Church; it was created by an exorcist priest and two young female mediums whose divine nature he revealed in 2009. The context in which this movement was born is one where after twenty years of a return to democracy, the population of Benin was confronted with failure to replace the political elite with fresh candidates, and with corruption and regionalism. This movement is not yet legally recognized and still too recent to be considered a new church; the majority of its followers belong to an urban youth who have expectations, yet lack opportunity to progress in society.

\section{Mots clés}

église catholique - Bénin - sorcellerie - institutions financières - corruption - personnel politique, non renouvellement.

\section{Keywords}


Catholic Church - Benin - vodun - witchcraft and sorcery - financial institutions - corruption - political staff, non renewal.

\section{Biographie}

E. Kadya Tall est anthropologue. Elle est chercheur à l'Institut de recherche pour le développement. . Ses recherches portent sur les productions culturelles de l'Atlantique Sud, les religions et le patrimoine. Elle est l'auteur de Le candomblé de Bahia, miroir baroque des mélancolies postcoloniales (2012).

\section{Biography}

E. Kadya Tall, an anthropologist, is Senior Researcher at the Institut de Recherche pour le Développement-Institut des mondes africains, Paris. Her works focuses on the cultural productions of the South Atlantic world, religions and heritage. She is the author of Le candomblé de Bahia, miroir baroque des mélancolies postcoloniales (2012).

\section{Index}

Adjovi, Séverin, 7

Amoussou, Bruno, 7

Banque mondiale, 9

Benoît XVI, 11, 13

Boni, Yayi, 6, 8, 9, 11, 17

capitalisme néolibéral, 8

cardinal de Souza, 2

christianismes africains, 2

clientélisme, 1, 10

Conférence nationale, $1,2,4,5,8,10,11$

Dieu, 1, 4, 5, 8, 9, 10, 11, 12, 13, 15, 16, 17

dimension théologico-politique, 5

Église de Banamè, 12, 13

Église du christianisme céleste, 2, 3

Église universelle du royaume de Dieu, 3

Églises congolaises, 16

ethos, 11

finance, 2,8

financiarisation, 4, 11, 12

FMI (Fond monétaire international), 4

Forces cauris pour un Bénin émergent, 5

géomancie $\mathrm{Fa}, 6$
Guezo, 6

Houngbédji, Adrien, 7

Kérékou, Mathieu, 4, 6

Kimpa Vita, 2

LEPI (liste électorale permanente informatisée), 7, 9

Lisa, 6

néo-pentecôtisme, 3

népotisme e, 1, 4, 7

père Aupiais, 2

pluralité religieuse, 2

programme d'ajustement structurel, 4

Renouveau charismatique, 14

Renouveau démocratique, 1, 4

Saint Étienne, 15, 16

Sainte Vierge, 1, 12, 13, 15, 17

Soglo, Nicéphore, 6, 8, 9

Soglo, Nicole, 1, 3, 12, 13

sorcellerie, 1, 2, 3, 5, 12, 13, 14, 16

Union fait la nation, 5

Vigan, Mathias, 12, 13

vodun, 5, 6, 7, 9, 13, 15, 16

Zo, 16 